\title{
Immune function of Chinese formula Qingwen Baidu granule in broilers
}

\author{
SHIJIN GUO ${ }^{l}$, SHIJUN FU ${ }^{l}$, QIANQIAN XU ${ }^{l}$, ZHIMEI ZHANG ${ }^{2}$, YANPING WANG ${ }^{2}$, \\ ZHIQIANG SHEN ${ }^{I}$
}

${ }^{1}$ Shandong Binzhou Animal Science and Veterinary Medicine Academy, Shandong, China

${ }^{2}$ Shandong Lvdu Ante Veterinary Drug Co. Ltd., Shandong, China

\begin{abstract}
This study was to investigate the effects of Qingwen Baidu granules on the antibody level, immune organ index and the lymphocyte transformation of broilers. Hy-line variety white cocks of 30 days were used to evaluate the antibody titer of Newcastle Disease in each serum group, and MTT method was used to determine the Tlymphocyte proliferation, and organ weighing methods to measure the immune organ index 21 days after immunization. The results showed that Qingwen Baidu granules could prolong the residue time in the body, improve the lymphocyte conversion ratio, increase the bursa, thymus and spleen index and promote immune organ development. These results suggested that Qingwen Baidu granules could improve the serum Newcastle disease antibody level, improve peripheral blood lymphocyte proliferation, enhance the cellular immune function, and elevate the immune organ index and growth, in order to raise the immune function in chicken. The above demonstrates that the Qingwen Baidu granules have significant effects on the cytoimmunity and humoral immunity, and the potentiation of the immune function in broilers.
\end{abstract}

Key words: Chinese formula Qingwen Baidu granule, immune function, broiler.

(Centr Eur J Immunol 2015; 40 (2): 149-152)

\section{Introduction}

Qingwen Baidu Yin was created by Yu Shi-yu of Qing Dynasty, who was a famous Epidemic Febrile Disease scholar, and included in "Yizhenyide". Qingwen Baidu prescription, was made up of 14 traditional Chinese medicines, which were Plaster, Rehmannia, Cornu bubali, Coptidis rhizome, Gardenia augusta Merr., Cortex moutan, Scutellaria, Paeoniae radix, Figwort root, Anemarrhenae, Forsythia suspense, Platycodon root, Glycyrrhiza uralensis and Lophatherum. It has been used in clinical medicine for more than two hundred years, and had a significant therapeutic effect to heal hair spot and high fever coma. The prescription was a complex of baihu decoction, huanglian jiedu decoction, cornus rhinoceri and rehmanniae decoction, the herbs have unity characters and effect on all aspects, and used for antipyretic detoxicate, clearing heat-fire and cooling blood [1]. The modern researches reported that Qingwen Baidu Yin inhibits hyper-immunological response in the prophase of sepsis, decreases impairment caused by hyper-immunological response [2], improves the cellular immunity level [3, 4], and bacteriostatic activity [5]. Qingwen baidu powder possesses significant preventive and cure effects on Pullorum disease and porcine reproductive and respiratory syndrome. Based on
Qingwen Baidu Yin, Qingwen Baidu granules were prepared by extracting main components separately using the scientific technology in this study. Systemic studies were mainly focused on the effects of Qingwen Baidu granules on the antibody titer, lymphocyte transformation as well as the immune organ index of chicken. These results could support the theory basis and practical guidance for clinical application and production.

\section{Material and methods}

\section{Animals}

Experimental procedures and protocols used in this study were approved by the Ethical committee of the Shandong Agricultural University of Veterinary, China, and conform to the "Guidelines for the care and use of laboratory animals" ( $8^{\text {th }}$ edition).

Ninety nonimmune Hy-line variety white cocks of 30 days were provided by the Poultry Institute of the Shandong Agricultural Academy. The animals were separated into 3 groups at random, 30 cocks per group, and brooding cages of 5 layers were used for breeding, and animals had free access to tap water and forage. The animals were fed

Correspondence: Shijun Fu, PhD, Shandong Binzhou Animal Science and Veterinary Medicine Academy, Shandong 256600, China, e-mail: fsj311@163.com 
with standard diet (Poultry Institute of the Shandong Agricultural Academy, China).

\section{Drugs}

Qingwen Baidu granules (batch number: 20120210, $200 \mathrm{~g} /$ package, $1 \mathrm{~g}$ granules equal to $1.3 \mathrm{~g}$ crude herbals) were provided by Shandong Lvdu Ante Veterinary Drug Co., Ltd. Newcastle Disease Vaccine, Live (Clone 30) is produced by Shandong Lvdu Bio-sciences and technology Co., Ltd. RPMI1640 medium, ConA (Sigma Chemical Co., St. Louis, MO, USA); MTT (AM-RESCO Co.); Lydroxypropylmethyl Cellulose (Shanghai Huajing Bio-sciences and technology Co., Ltd.); $10 \%$ fetal bovine serum (Gibco BRL, New York, USA); SDS (Sigma Chemical Co., St. Louis, MO, USA); Heparin Sodium (Ao Box Biotechnology Co., Ltd.).

\section{Material and methods}

The animals were separated into 3 groups: test drug group animals were administered Qingwen Baidu granules by drinking water at $0.5 \mathrm{~g} / \mathrm{l}$ dosage for 3 days; a positive control group was treated with levamisole (1\%) for 3 days and a negative control group without any treatment. After 3 days' treatment, two times a dosage of Newcastle Disease Vaccine, Live (Clone 30) was administered by eye drops or nasal inhalation.

Ten chickens were randomly chosen for sterile blood collection at wing veins on the immune day and 7, 14 and 21 days later, serum was separated, preserved in the low temperature refrigerator, and used for Newcastle disease antibodies determination. HI valence was determined by the micro-hemagglutination-inhibition test [6].

Lymphocyte transformation assay [7, 8]: $10 \mathrm{ml}$ blood from chicken hearts were sterile collected, $1 \mathrm{ml}$ heparin was used for anticoagulation, and placed in the super clean bench, and then $10 \mathrm{ml}$ RPMI 1640 medium (contains $10 \%$ fetal bovine serum) were added, after mixing, the sample was separated into 5 portions of $4 \mathrm{ml}$ per portion. The blood sample was subsequently imposed on the upper tier of $4 \mathrm{ml} \mathrm{Ly}$ droxypropylmethyl cellulose along the centrifuge tube wall carefully, then centrifuged at $2000 \mathrm{r} / \mathrm{min}$ for 20 min, and assimilated the white lymphocyte layer into another sterile centrifuge tube, 2-3 ml RPMI 1640 medium were added and centrifuged at $2000 \mathrm{r} / \mathrm{min}$ for $20 \mathrm{~min}$ again, two more times were needed, and followed with centrifugation. After $0.6 \%$ trypan blue staining, the live lymphocyte was counted and using serum free RPMI 1640 medium to adjust cell concentration at $4 \times 10^{6}$ cells $/ \mathrm{ml}$.

Lymphocytes culture and measurement: $50 \mu \mathrm{l}$ per well of RPMI 1640 medium (contains 10\% fetal bovine serum, $5 \mathrm{~g} / \mathrm{ml}$ ConA) were dipped into 96-well cell culture plates, coupled with 50 micro liter lymphocytes suspension, and no-cell RPMI1640 medium was used as zero control. Cell culture plates were incubated at $\mathrm{CO}_{2}$ incubator $\left(39^{\circ} \mathrm{C}\right.$ in $5 \% \mathrm{CO}_{2}$ and saturated humidity) for $66 \mathrm{~h}$. In the super clean bench, 151 per well of $5 \mathrm{mg} / \mathrm{ml}$ MTT were added, followed by $4 \mathrm{~h}$ incubation, and $10 \%$ SDS- $0.01 \mathrm{~mol} / 1 \mathrm{HCl}$ 1001 per well were added, then $2 \mathrm{~h}$ incubation and the plates were put at room temperature for $5 \mathrm{~min}$. The OD570 was determined with T6 new millennium ultraviolet-visible spectrophotometer.

Immune organ index measurement: 10 chickens were randomly selected for killing, 20 days after immunization; the organ weight measure method was used to determine bursa fabricii, thymus gland and spleen index.

$$
\text { immune organ index }=\frac{\text { immuneorgan weight }(\mathrm{mg})}{\text { bodyweight }(\mathrm{g}) \times 100 \%} \times 100 \%
$$

\section{Statistics}

Data are presented as means \pm SEM; data were analyzed with ANOVA by SPSS 17.0 software. Differences were considered significant at $p<0.05$.

\section{Results}

\section{Valence of Newcastle disease antibody measurement in chickens}

Table 1 shows that the titre of the Newcastle disease antibody of test drug group chickens was significantly higher than those of the positive control group and the negative control group $(p<0.05)$; there was no significant difference between the positive control group and the negative control group $(p>0.05)$. These results indicate that the Qingwen Baidu granules could enhance the humoral immunity in chicken.

Table 1. Influence of Qingwen Baidu Granules to fowl Newcastle disease antibody titer

\begin{tabular}{|c|c|c|c|c|c|c|}
\hline \multirow[b]{2}{*}{ Test days } & & \multicolumn{3}{|c|}{ Numbers } & \multicolumn{2}{|l|}{ Titre $(\log 2)$} \\
\hline & & $\mathbf{0}$ & $7 \mathrm{~d}$ & $14 \mathrm{~d}$ & $21 \mathrm{~d}$ & $28 \mathrm{~d}$ \\
\hline Test drug group & 10 & 0 & $6.54 \pm 0.58^{a}$ & $8.73 \pm 0.68^{a}$ & $8.23 \pm 0.76^{\mathrm{a}}$ & $7.99 \pm 0.74^{\mathrm{a}}$ \\
\hline Positive control group & 10 & 0 & $4.62 \pm 0.73^{b}$ & $6.96 \pm 0.85^{b}$ & $6.35 \pm 0.72^{b}$ & $5.87 \pm 0.93^{b}$ \\
\hline Negative control group & 10 & 0 & $4.54 \pm 0.41^{b}$ & $6.82 \pm 0.77 b$ & $6.31 \pm 0.65^{b}$ & $5.79 \pm 0.66^{\mathrm{b}}$ \\
\hline
\end{tabular}

Values with superscripts in the same column mean not significant $p>0.05$; while those with different ones mean a significant difference of $p<0.05$. 
Table 2. Influence of Qingwen Baidu Granules to fowl total T-cellular score

\begin{tabular}{lccccc}
\hline Groups & Repeat 1 & Repeat 2 & Repeat 3 & Repeat 4 & \pm SD \\
\hline Test drug group & 0.144 & 0.155 & 0.153 & 0.149 & $0.150 \pm 0.0049^{\mathrm{a}}$ \\
\hline Positive control group & 0.136 & 0.142 & 0.151 & 0.137 & $0.142 \pm 0.0069^{\mathrm{a}}$ \\
\hline Negative control group & 0.039 & 0.037 & 0.036 & 0.035 & $0.037 \pm 0.0017^{\mathrm{b}}$ \\
\hline Values with superscripts in the same column mean not significant $p>0.05 ;$ while those with different ones mean a significant difference of $p<0.05$.
\end{tabular}

Table 3. Influence of Qingwen Baidu Granules to fowl immune organ index (\%)

\begin{tabular}{lccc}
\hline Groups & Bursa fabricii index & Spleen index & Thymus index \\
\hline Test drug group & $4.52 \pm 0.79^{\mathrm{a}}$ & $2.54 \pm 0.47$ & $3.37 \pm 0.75^{\mathrm{a}}$ \\
\hline Positive control group & $3.87 \pm 0.62^{\mathrm{b}}$ & $2.48 \pm 0.41^{\mathrm{b}}$ & $2.99 \pm 0.47^{\mathrm{b}}$ \\
\hline Negative control group & $3.79 \pm 0.57^{\mathrm{b}}$ & $2.12 \pm 0.29^{\mathrm{b}}$ & $2.91 \pm 0.40^{\mathrm{b}}$ \\
\hline Values with superscripts in the same column mean not significant $p>0.05 ;$ while those with different ones mean a significant difference of $p<0.05$.
\end{tabular}

\section{Measurement of peripheral blood T lymphocyte}

Table 2 shows that the test drug group and the positive control group both improved the lymphocyte conversion ratio $(p<0.05)$, and enhanced the cellular immune function. These results indicated that the Qingwen Baidu granules could raise the disease resistance of broilers.

\section{Immune organ index measurement}

Bursa fabricii, thymus gland and spleen index of the test drug group were significantly higher than those of the positive control group and the negative control group $(p<0.05)$ (Table 3). These results confirmed that the Qingwen Baidu granules could enhance the immune function.

\section{Discussion}

The serum antibody level is one of the most important indexes to reflect the humoral immunity level. The high serum antibody level generally means the stronger resistance of the organism to certain diseases. In this study, the Qingwen Baidu Granules showed the significant influence on the Newcastle disease antibody titer. The antibody titer descended slowly after $21 \mathrm{~d}$ immunization and prolonged the remaining time. $T$ lymphocytes mainly mediated the cellular immunity, and the T lymphocytes proliferation is one of the most important indexes to evaluate the cellular immune function. MTT method was adopted to measure the OD value, and the results showed the OD value was the direct proportion to lymphocytes proliferation ratios. These results indicated that the Qingwen Baidu granules could significantly improve the lymphocyte conversion ratio.

Thymus gland, bursa of Fabricius and spleen are important immune organs of broilers. Generally, cell growth, development and division caused animal immune organ weight increase, and the immune organ weights reflected the immune function. Accordingly, the elevation of the im- mune organ index indicates the maturation of the immune system. In this study, immune organ indexes were measured, and the results showed that Qingwen Baidu granules can significantly improve the Bursa fabricii, thymus gland and spleen indexes, and promote the immune organ growth, and enhance specific and non-specific immunity. It was reported that polysaccharides from Platycodon grandiflorum A. DC had the obvious immune-improving effects on immunosuppressive mice induced by CTX; Scrophularia ningpoensis hemsl could improve the serum antibody level in mice [9]; Glycyrrhiza polysaccharide could promote serum hemolysin IgM and IgG production, increase the antibody-production cell level, enhance the macrophage engulfing carbon granula ability and improve the thymus and spleen index [10]; Cortex moutan polysaccharides PSM2b could enhance T lymphocytes function, and activate macrophages [11].

In conclusion, the Chinese Qingwen Baidu granules can improve the serum Newcastle Disease antibody level, improve peripheral blood lymphocyte proliferation, enhance cellular immune function and elevate the immune organ index and growth.

This work was financially supported by the Shandong Modern Agricultural Technology \& Industry System, China (SDAIT-13-011-10).

The authors declare no conflict of interest.

\section{References}

1. Leng JC, Wang WX (2010): Influence of Qingwen baidu decoction on clinical therapeutic effect against sepsis and seroimmunity indexes. J Emerg Trad Chin Med 9: 1483-4184. 
2. Li SM, Yu LL (2010): Action mechanism of Qingwei Baidu decoction in treatment of fever period of hemorrhagic fever with renal syndrome. Heilongjiang Med Pharm 6: 23-24.

3. Ning GB, Zheng MX, Wen WY (1999): In vitro bacteriostasis test of Qingwen Baidu powder and Xie Li Kang. Chin J Vet Med 2: 56-58.

4. Ning GB, Zheng MX, Wen WY (1998): Control efficiency of Qingwen Baidu powder against Pullorum disease. Chin J Vet Med 12: 34.

5. Tang YP, Zhou SH (2008): Control efficiency of three Chinese drugs against high pathogenicity porcine reproductive and respiratory syndrome. Livestock and Poultry Industry 3: 53-54.

6. Cui ZZ (2006): Veterinary Immunology Laboratory Procedure. China Agricultural Press, Beijing.

7. Yang FL, Yue H, Luo W, et al. (2002): Research on optimum condition of detecting chicken peripheral lymphocyte proliferate response by MTT assay. J Southwest University for Nationalities 2: 214-215.

8. Zheng TS, Wang GP, Hu YL, et al. (2010): Influence of compound traditional recipe on cell proliferation of peripheral blood T lymphocytes in chickens. Animal Husbandry and Veterinary Medicine 2: 110-111.

9. Jia L, Lu JJ, Zhou WY, et al. (2012): Immune regulatory effect of polysaccharides from platycodom grandiflorum on immunosuppressive mice induced by cyclophosphamide. Food and Machinery 3: 112-114.

10. Hu J, Ao MZ, Cui YM (2008): Anti-tumor effect of polysaccharide from glycyrrhizin and its influence on immunological function. Natural Product Research and Development 5: 911913.

11. Dai L, Zhao ZP, Shen YS, et al. (2001): Effects of polysaccharide of Mudan Cortex PSM2b on immune functions of mice in vitro. Acta Laser Biology Sinica 1: 48-50. 